Advances in Dynamical Systems and Applications (ADSA).

ISSN 0973-5321, Volume 15, Number 2, (2020) pp. 271-281

(C) Research India Publications

https://dx.doi.org/10.37622/ADSA/15.2.2020.271-281

\title{
A New Version of the Minimax Problem
}

\author{
Azennar Radouane ${ }^{* 1}$, Fouad Ouzine ${ }^{\dagger 2}$, and Driss Mentagui ${ }^{\ddagger 3}$ \\ 1,2,3 Department of Mathematics, Faculté des Sciences, Ibn, \\ Tofaïl University, B.P. 133, Kenitra, 14000, Morocco.
}

\begin{abstract}
In this paper, we establish a new type of minimax problem using the results given by [1].
\end{abstract}

2010 Mathematics Subject Classification: 47H08;54H25;49J35,46A03

Keywords: Fixed Point, measure of noncompactness, multivalued, ordered locally convex spaces, game theory

\section{INTRODUCTION}

Questions concerning the minimax problems have been studied extensively in the literature. These questions are important because the minimax theorem offers a wide array of applications. Minimax theorems of real-valued functions have been discussed since the 1960s; see [7], [3], [8], [9], [13], [1] and references therein.

In recent years, based on the development of game theory, optimization theory, and economics, a great deal of research has been devoted to the study of the minimax theorem [17],[24], [26].

\footnotetext{
*E-mail: fouad.ouzine@uit.ac.ma

†E-mail: radouane.azennar@uit.ac.ma

${ }_{\ddagger}^{\ddagger}$ E-mail: dri_mentagui@yahoo.fr
} 
A lot of research has been devoted to the study of the existence of a fixed points of single-valued and multivalued mappings in ordered Banach spaces [2],[19], and in complete locally convex spaces [11], [12]. In this paper, we establish some fixed point theorems of multivalued mappings a complete ordered locally convex spaces under weaker assumptions.

It is well known that partial order plays an important role in optimization theory. The optimization problems in the previous references are studied in the partial order induced by a closed cone such as the subsets of space are compact convex or closed convex. But in some situations, the subsets of space are order convex.

The object of this paper is to present a new versions of the Minimax Problem.

\section{NOTATIONS AND PRELIMINARIES}

Let $E$ be a real vector space. A cone $K$ in $E$ is a subset of $E$ with $K+K \subset K$, $\alpha K \subset K$ for all $\alpha \geq 0$, and $K \cap(-K)=\{0\}$. As usual $E$ will be ordered by the (partial) order relation

$$
x \leq y \Leftrightarrow y-x \in K
$$

and the cone $K$ will be denoted by $E^{+} . E$ is said to be an ordered topological vector space, if $E$ is an ordered vector space equipped with a linear topology for which the positive cone $E^{+}$is closed. For two vectors $x, y \in E$ the order interval $[x, y]$ is the set defined by

$$
[x, y]=\{z \in E: x \leq z \leq y\} .
$$

Note that if $x \notin y$ then $[x, y]=\phi$.

A cone $E^{+}$of an ordered topological vector space $E$ is said to be normal whenever the topology of $E$ has a base at zero consisting of order convex sets. If the topology of $E$ is also locally convex, then $E$ is said to be an ordered locally convex space, and in this case the topology of $E$ has a base at zero consisting of open, circled, convex, and order convex neighborhoods.

$\overline{\operatorname{conv}}(A)$ denotes the closed convex hull of $A$.

The following two lemmas will be useful in the proofs of our results.

Lemma 2.1 ([4, Lemma 2.3]). If $E$ is an ordered topological vector space, then $E$ is Hausdorff and the order intervals of E are closed.

Lemma 2.2 ([4, Lemma 2.22 and Theorem 2.23]). If the cone $E^{+}$of an ordered topological vector space $(E, \tau)$ is normal, then the following assertions hold:

\section{Every order interval is $\tau$-bounded.}


2. For every two nets $\left(x_{\alpha}\right),\left(y_{\alpha}\right) \subset E$, (with the same index set I) satisfy $0 \leq x_{\alpha} \leq$ $y_{\alpha}$ for each $\alpha$ and $y_{\alpha} \stackrel{\tau}{\rightarrow} 0$ imply $x_{\alpha} \stackrel{\tau}{\rightarrow} 0$.

Let $E$ be an ordered locally convex space whose topology is defined by a family $\mathcal{P}$ of continuous semi-norms on $E, \mathcal{B}$ is the family of all bounded subsets of $E$, and $\Phi$ is the space of all functions $\varphi: \mathcal{P} \rightarrow \mathbb{R}^{+}$with the usual partial ordering $\varphi_{1} \leq \varphi_{2}$ if $\varphi_{1}(p) \leq \varphi_{2}(p)$ for all $p \in \mathcal{P}$. The measure of noncompactness on $E$ is the function $\alpha: \mathcal{B} \rightarrow \Phi$ such that for every $B \in \mathcal{B}, \alpha(B)$ is the function from $\mathcal{P}$ into $\mathbb{R}^{+}$defined by

$$
\alpha(B)(p)=\inf \left\{d>0: \sup \left\{p(x-y): x, y \in B_{i}\right\} \leq d \forall i\right\}
$$

where the infimum is taken on all subsets $B_{i}$ such that $B$ is finite union of $B_{i}$. Properties of measure of noncompactness in locally convex spaces are presented in [11, Proposition 1.4].

An operator $T: Q \subset E \rightarrow E$ is called to be countably condensing if $T(Q)$ is bounded and if for any countably bounded set $A$ of $Q$ with $\alpha(A)(p)>0$ we have

$$
\alpha(T(A))(p)<\alpha(A)(p)
$$

Definition 2.3. Let $E$ be a complete ordered locally convex space with a normal cone $E^{+}$. An element $x \in E$ is said to be a fixed point of a multivalued mapping $T: E \rightarrow 2^{E}$ if $x \in T(x)$.

Definition 2.4. Let $E$ be a complete ordered locally convex space with a normal cone $E^{+}$. Let $A, B \in 2^{E}$. Then $A \leq B$ means $a \leq b$ for all $a \in A$ and $b \in B$.

A map $T: E \rightarrow 2^{E}$ is said to be isotone nondecreasing if for $x, y \in E$ and $x \leq y$ we have $T x \leq T y$.

A map $T: E \rightarrow 2^{E}$ is said to be isotone nonincreasing if for $x, y \in E$ and $x \leq y$ we have $T x \geq T y$.

Definition 2.5. Let $E$ be a complete ordered locally convex space with a normal cone $E^{+}$, let $Q \subset E$, an operator $T: Q \rightarrow 2^{Q}$ is called to be countably condensing if $T(Q)$ is bounded and if for any countably bounded set $A$ of $Q$ with $\alpha(A)(p)>0$ we have

$$
\alpha(T(A))(p)<\alpha(A)(p)
$$

with $T(A)=\cup_{x \in A} T x$

Lemma 2.6. Let $E$ be an ordered topological vector space with a normal cone $E^{+}$. Then a monotone net $\left(u_{\alpha}\right) \subset E$ is convergent if and only if it has a weakly convergent subnet. 
Lemma 2.7. Let $E$ be a complete locally convex space. Let $C \subset E$ be a nonempty closed,bounded and convex subset, and let $T: C \rightarrow 2^{C}$ be a condensing map, Then there exists a nonempty compact convex subset $K$ of $E$ such that $T(K) \subset K$.

Theorem 2.8. [18] Let $E$ be a complete locally convex space. Let $C \subset E$ be a nonempty compact convex subset, and let $T: C \rightarrow 2^{C}$ be a monotone closed such that $T(x)$ is nonempty closed and convex for every $x \in C$. Then :

$T$ has a fixed point $u \in C$.

The following theorem is the basic theorem of [1].

Theorem 2.9. Let $E$ be a complete ordered locally convex space with a normal cone $E^{+}$. Let $\Omega$ be an order convex subset of $E$, and let $u_{0}, v_{0} \in \Omega, u_{0} \leq v_{0}$ and let $T: \Omega \rightarrow 2^{\Omega}$ be a monotone closed and isotone nonincreasing mappings such that $T^{2}$ fixes the interval $\left[u_{0}, v_{0}\right]$ : Suppose that $T$ is condensing and $T(x)$ is nonempty closed and convex for every $x \in C$.

Then, $T$ has a fixed point in $\Omega$.

See [1] for more details.

\section{MAIN RESULTS}

The following results generalize the results of [1] in complete ordered locally convex spaces whith low condtions.

In this section we will give an application of theorem 2.9 to game theory with a new method and new hypothesis.

A game is a triple $(A, B, K)$, where $A, B$ are nonempty ordred sets ([17, page 326] ), whose elements are called strategies, and $K: A \times B \rightarrow \mathbb{R}$ is the gain function. There are two players, $\alpha$ and $\beta$, and $K(x, y)$ represents the gain of the player $\alpha$ when he chooses the strategy $x \in A$ and the player $\beta$ chooses the strategy $y \in B$. The quantity $-K(x, y)$ represents the gain of the player $\beta$ in the same situation. The target of the player $\alpha$ is to maximize his gain when the player $\beta$ chooses a strategy that is the worst for $\alpha$, that is, to choose $x_{0} \in A$ such that :

$$
\inf _{y \in B} K\left(x_{0}, y\right)=\max _{x \in A} \inf _{y \in B} K(x, y) .
$$

Similarly, the player $\beta$ chooses $y_{0} \in B$ such that:

$$
\sup _{x \in A} K\left(x, y_{0}\right)=\min _{y \in B} \sup _{x \in A} K(x, y) .
$$


It follows

$$
\sup _{x \in A} \inf _{y \in B} K(x, y)=\inf _{y \in B} K\left(x_{0}, y\right) \leq K\left(x_{0}, y_{0}\right) \leq \sup _{x \in A} K\left(x, y_{0}\right) \leq \inf _{y \in B} \sup _{x \in A} K(x, y) .
$$

Note that in general

$$
\sup _{x \in A} \inf _{y \in B} K(x, y) \leq \inf _{y \in B} \sup _{x \in A} K(x, y) .
$$

If the equality holds in (4.3), then, by (4.4),

$$
\sup _{x \in A} \inf _{y \in B} K(x, y)=K\left(x_{0}, y_{0}\right)=\inf _{y \in B} \sup _{x \in A} K(x, y) .
$$

The common value in (4.5) is called the value of the game, $\left(x_{0}, y_{0}\right) \in A \times B$ a solution of the game and $x_{0}$ and $y_{0}$ winning strategies. It follows that to prove the existence of a solution of a game we have to prove equality (4.4). For more details on game theory and minimax theorems, we refer to the books of Aubin, J.P [24] and Carl, S, Heikkilä, S [17].

Let $\left(X, \leq_{X}\right)$ and $\left(Y, \leq_{Y}\right)$ be a complete ordered locally convex spaces, Consider in the product space $(X \times Y, \ll)$ the following partial orders:

For any $A_{1} \times B_{1}, A_{2} \times B_{2} \in 2^{X \times Y}$, we denote :

$$
A_{1} \times B_{1} \ll A_{2} \times B_{2} \text { iff } A_{1} \leq_{X} A_{2} \text { and } B_{1} \leq_{Y} B_{2}
$$

With

$$
A_{1} \leq_{X} A_{2} \text { iff } x_{1} \leq_{X} x_{2}, \quad \forall\left(x_{1}, x_{2}\right) \in A_{1} \times A_{2},
$$

It is easy to see that if $X^{+}$is a normal cone in $X$ and $Y^{+}$is a normal cone in $Y$, then $\left(X^{+} \times Y^{+}\right)$is also a normal cone of the product ordered topological space $X \times Y$. In this section, $\leq$ and $<$ mean the total order relation of $\mathbb{R}$.

$$
\phi(x)=\min _{y \in B} K(x, y)=\min K(x \times B), x \in A
$$

and

$$
\psi(y)=\max _{x \in A} K(x, y)=\max K(A \times y), y \in B
$$

We exclude the trivial case, and we will assume that the sets :

$$
\{x \in A: K(x, y)=\psi(y)\} \text { and }\{y \in B: K(x, y)=\phi(x)\}
$$

are nonempty.

We set that :

$$
N_{y}=\{x \in A: K(x, y)=\psi(y)\} \text { and } M_{x}=\{y \in B: K(x, y)=\phi(x)\}
$$


In the sequel, we consider the measure of noncompactness $\alpha^{\times}(p)$ on a product of locally convex spaces. See [28].

Theorem 3.1. Let $X_{1}, X_{2}, \ldots, X_{n}$ be a complete locally convex spaces. Assume that $\alpha_{1}, \alpha_{2}, \ldots, \alpha_{n}$ be the measure of noncompactness in $X_{1}, X_{2}, \ldots, X_{n}$ respectively, Suppose $F:\left(\left[0,+\infty[)^{n} \rightarrow[0,+\infty[\right.\right.$ is :

\section{Convexe.}

2. $F\left(x_{1}, x_{2}, \ldots, x_{n}\right)=0$ if and only if $x_{i}=0$ for $i=1,2, \ldots, n$.

then, for each $D \in \mathcal{B}\left(X_{1} \times X_{2} \times \ldots \times X_{n}\right)$ :

$$
\alpha^{\times}(D)(p)=F\left(\alpha_{1}\left(D_{1}\right)(p), \alpha_{2}\left(D_{2}\right)(p), \ldots, \alpha_{n}\left(D_{n}\right)(p)\right) .
$$

defines a measure of noncompactness in $X_{1} \times X_{2} \times \ldots \times X_{n}$ where $D_{1}, D_{2}, \ldots, D_{n}$ denote the natural projection of $D$ into $X_{i}$ for $i=1, \ldots, n$.

Lemma 3.2. [10, Lemma 3.3.] If $A, B$ are compact Hausdorff topological spaces and $K: A \times B \rightarrow \mathbb{R}$ is continuous, then the functions :

$$
\phi(x)=\min _{y \in B} K(x, y)=\min K(x \times B), x \in A
$$

and

$$
\psi(y)=\max _{x \in A} K(x, y)=\max K(A \times y), y \in B
$$

are continuous too.

Theorem 3.3. Let $\left(X, \leq_{X}\right)$ and $\left(Y, \leq_{Y}\right)$ be a complete ordered locally convex spaces, let $A \subset X$ and $B \subset Y$ nonempty order convex sets.

Let $c, c^{\prime} \in A \times B$ such that $c \ll c^{\prime}$.

Suppose that :

1. The functions $K: A \times B \rightarrow \mathbb{R}$ is continuous.

2. $\forall x, x^{\prime} \in A$ with $x \leq_{X} x^{\prime} \Rightarrow M_{x} \geq_{Y} M_{x^{\prime}}$, and $\forall y, y^{\prime} \in B$ with $y \leq_{Y} y^{\prime} \Rightarrow N_{y} \geq_{X} N_{y^{\prime}}$

3. $T: A \times B \rightarrow 2^{A \times B}$ defined as $T(x, y)=N_{y} \times M_{x}$ is $\alpha^{\times}$-condensing.

4. $T^{2}$ fixes the order interval $\left[c, c^{\prime}\right]$

5. for every $x \in A$, the function $K(x,$.$) is convex.$ 
6. for every $y \in B$ the function $K(., y)$ is concave.

Then,

$$
\min _{y \in B} \max _{x \in A} K(x, y)=\max _{x \in A} \min _{y \in B} K(x, y)
$$

and the game $(A, B, K)$ has a solution.

Proof. We pose : $C=A \times B$ and $c=(x, y)$.

the product set $C$ is ordred convex,(product of two ordred convex).

Since $T: C \rightarrow 2^{C}$ is $\alpha^{\times}$-condensing, it follows from Lemma 2.7 that there exists a compact convex subset $Q$ of $C$ such that $T(Q) \subset Q$.

It is easy to see that if $Q$ is ordred convex.

therefore, the following two mapping can be defined by:

$$
\begin{aligned}
T: Q & \rightarrow 2^{Q} \\
& c \mapsto N_{y} \times M_{x}
\end{aligned}
$$

First, we will show that $T$ have the closed graph.

Indeed, Let $\left\{\left(x_{\alpha}, y_{\alpha}\right)\right\}$ be a net in $Q$ such that $\left(x_{\alpha}, y_{\alpha}\right) \rightarrow(x, y) \in Q$, let $\left\{\left(u_{\alpha}, v_{\alpha}\right)\right\}$ be a net such that $\left(u_{\alpha}, v_{\alpha}\right) \in T\left(x_{\alpha}, y_{\alpha}\right)$,

and $\left(u_{\alpha}, v_{\alpha}\right) \rightarrow(u, v)$, We shall show that $(u, v) \in T(x, y)$, we have :

$$
\begin{aligned}
\left(u_{\alpha}, v_{\alpha}\right) \in T\left(x_{\alpha}, y_{\alpha}\right) & \Leftrightarrow\left(u_{\alpha}, v_{\alpha}\right) \in N_{y_{\alpha}} \times M_{x_{\alpha}} \\
& \Leftrightarrow K\left(u_{\alpha}, y_{\alpha}\right)=\psi\left(y_{\alpha}\right) \text { and } K\left(x_{\alpha}, v_{\alpha}\right)=\phi\left(x_{\alpha}\right)
\end{aligned}
$$

Since $K$ and $\phi$ are continuous (See Lemma3.2), for $\alpha \in I$, we will have that :

$$
K(u, y)=\psi(y) \text { and } K(x, v)=\phi(x)
$$

So, $(u, v) \in T(x, y)$, which implies that $T$ has a closed graph.

Using hypothesis (2), it is clear to see that $T$ is nonincreasing and By hypothesis (4), we have $T^{2}$ fixed the interval $\left[c, c^{\prime}\right]$.

Finally, Using hypothesis (5) and (6), it is clear to see that $N_{y}$ and $M_{x}$ are convex too, so, $T(c)$ is nonempty closed and convex for every $c \in C$

Thus, by theorem 2.9 ,

$T$ has a fixed point $c^{\star}=\left(x^{\star}, y^{\star}\right)$.

So, we have $c^{\star} \in T c^{\star}=N_{y^{\star}} \times M_{x^{\star}}$.

in other words,

$$
x^{\star} \in N_{y^{\star}} \Leftrightarrow K\left(x^{\star}, y^{\star}\right)=\max _{x \in A} K\left(x, y^{\star}\right) \geq \inf _{y \in B} \max _{x \in A} K(x, y)
$$




$$
y^{\star} \in M_{x^{\star}} \Leftrightarrow K\left(x^{\star}, y^{\star}\right)=\min _{y \in B} K\left(x^{\star}, y\right) \leq \sup _{x \in A} \min _{y \in B} K(x, y)
$$

Taking into account these last two inequalities and (4.4), we get

$$
K\left(x^{\star}, y^{\star}\right) \leq \sup _{y \in B} \min _{x \in A} K(x, y) \leq \inf _{x \in A} \max _{y \in B} K(x, y) \leq K\left(x^{\star}, y^{\star}\right)
$$

implying

$$
\max _{x \in A} \min _{y \in B} K(x, y)=K\left(x^{\star}, y^{\star}\right)=\min _{y \in B} \max _{x \in A} K(x, y)
$$

This completes the proof.

Corollary 3.4. Under the same assumptions of the theorem 3.3 and we now consider that:

$\exists\left(x_{0}, y_{0}\right),\left(x_{1}, y_{1}\right) \in X \times Y$ such that $\left(x_{0}, y_{0}\right) \ll\left(x_{1}, y_{1}\right)$ and The functions $K:\left[x_{0}, x_{1}\right] \times\left[y_{0}, y_{1}\right] \rightarrow \mathbb{R}$.

Then,

$$
\min _{y \in\left[y_{0}, y_{1}\right]} \max _{x \in\left[x_{0}, x_{1}\right]} K(x, y)=\max _{x \in\left[x_{0}, x_{1}\right]} \min _{y \in\left[y_{0}, y_{1}\right]} K(x, y)
$$

and the game $(X, Y, K)$ has a solution.

Proof. Since $\left[x_{0}, x_{1}\right]$ and $\left[y_{0}, y_{1}\right]$ are two ordred convex sets in $X$ and $Y$ respectively. therefore, we apply the previous theorem for $A=\left[x_{0}, x_{1}\right]$ and $B=\left[y_{0}, y_{1}\right]$.

We will give another application of the theorem 2.9 is to result of J.von Neumann [25] (see also [26]).

Theorem 3.5. Let $\left(X, \leq_{X}\right)$ and $\left(Y, \leq_{Y}\right)$ be a complete ordered locally convex spaces and $A \subset X, B \subset Y$ nonempty ordred convex sets.

Let $c, c^{\prime} \in A \times B$ such that $c \ll c^{\prime}$.

For $Q, Q^{\prime} \subset A \times B$, we pose :

$$
N_{y}=\{x \in A:(x, y) \in Q\} \text { and } M_{x}=\left\{y \in B:(x, y) \in Q^{\prime}\right\}
$$

Suppose that :

1. The sets $Q, Q^{\prime}$ are closed.

2. $\forall x, x^{\prime} \in A$ with $x \leq_{X} x^{\prime} \Rightarrow M_{x} \geq_{Y} M_{x^{\prime}}$, and $\forall y, y^{\prime} \in B$ with $y \leq_{Y} y^{\prime} \Rightarrow N_{y} \geq_{X} N_{y^{\prime}}$

3. $T: A \times B \rightarrow 2^{A \times B}$ defined as $T(x, y)=N_{y} \times M_{x}$ is $\alpha^{\times}$-condensing.

4. $T^{2}$ fixes the order interval $\left[c, c^{\prime}\right]$ 
5. for every $x \in A$, the function $K(x,$.$) is convex.$

6. for every $y \in B$ the function $K(., y)$ is concave.

then, $Q \cap Q^{\prime} \neq \emptyset$

Proof. We pose : $C=A \times B$ and $c=(x, y)$.

the product set $C$ is ordred convex,(product of two ordred convex)

therefore, the following two mapping can be defined by:

$$
\begin{aligned}
T: C & \rightarrow 2^{C} \\
c & \mapsto N_{y} \times M_{x}
\end{aligned}
$$

whith $c=(x, y) \in A \times B$.

First, we will show that $T$ have the closed graph.

Indeed, Let $\left\{\left(x_{\alpha}, y_{\alpha}\right)\right\}$ be a net in $C$ such that $\left(x_{\alpha}, y_{\alpha}\right) \rightarrow(x, y) \in C$, let $\left\{\left(u_{\alpha}, v_{\alpha}\right)\right\}$ be a net such that $\left(u_{\alpha}, v_{\alpha}\right) \in T\left(x_{\alpha}, y_{\alpha}\right)$,

and $\left(u_{\alpha}, v_{\alpha}\right) \rightarrow(u, v)$, We shall show that $(u, v) \in T(x, y)$,

we have :

$$
\begin{aligned}
\left(u_{\alpha}, v_{\alpha}\right) \in T\left(x_{\alpha}, y_{\alpha}\right) & \Leftrightarrow\left(u_{\alpha}, v_{\alpha}\right) \in N_{y_{\alpha}} \times M_{x_{\alpha}} \\
& \Leftrightarrow u_{\alpha} \in N_{y_{\alpha}} \text { and } v_{\alpha} \in M_{x_{\alpha}} \\
& \Leftrightarrow\left(u_{\alpha}, y_{\alpha}\right) \in Q \text { and }\left(x_{\alpha}, v_{\alpha}\right) \in Q^{\prime}
\end{aligned}
$$

Since $Q, Q^{\prime}$ are closed, for $\alpha \in I$, we will have that :

$$
(u, y) \in Q \text { and }(x, v) \in Q^{\prime}
$$

So, $(u, v) \in T(x, y)$, which implies that $T$ has a closed graph.

We proof the rest by the same method of theorem 3.3.

Thus, by theorm $2.9 T$ has a fixed point $c^{\star}=\left(x^{\star}, y^{\star}\right)$.

So, we have $c^{\star} \in T c^{\star}$.

in other words,

$$
\left(x^{\star}, y^{\star}\right) \in N_{y^{\star}} \times M_{x^{\star}} \Leftrightarrow x^{\star} \in N_{y^{\star}} \text { and } y^{\star} \in M_{x^{\star}}
$$

implying

$$
\left(x^{\star}, y^{\star}\right) \in Q \cap Q^{\prime}
$$

This completes the proof.

\section{Conflicts of Interest:}

Te authors declare that there are no conficts of interest regarding the publication of this paper. 


\section{REFERENCES}

[1] Azennar Radouane, Driss Mentagui, A solution for the minimax problem via fixed point theory in complete ordered locally convex spaces, Communications in Optimization Theory, Vol. 2020 (2020), Article ID 11, pp. 1-10

[2] D. Guo, V. Lakshmikantham, Nonlinear Problems in Abstract Cones, Academic Press, Boston, 1988.

[3] Ouzine Fouad, Azennar Radouane, Driss Mentagui, Common Coupled Fixed Point Results For Multivalued Mappings In Ordered Banach Spaces, Dynamic Systems and Applications 29 (2020) (In Press)

[4] C. D. Aliprantis, R. Tourky, Cones and duality, Graduate Studies in Mathematics, Volume 84, American Mathematical Society, Providence, RI, USA; 2007:xiv+279.

[5] K. Deimling, Nonlinear Functional Analysis. Springer-Verlag (1984).

[6] C.J.Himmelberg,J.R.Porter and F. S.Van Vleck,Fixed Point Theorems for Condensing Multifunctions,Proceedings of the American Mathematical Society, Vol. 23, No. 3 (Dec., 1969), pp. 635-641

[7] K. Fan, Minimax theorems, Proc. Natl. Acad. Sci. USA 39 (1953) 42-47.

[8] K. Fan, A minimax Inequality and Applications, Inequalities, III, Academic Press, New York, 1972.

[9] C.W. Ha, Minimax and fixed point theorems, Math. Ann. 248 (1980) 73-77.

[10] S.Cobzas, Fixed Point Theorems in Locally Convex Spaces- The Schauder Mapping Method. Hindawi Publishing Corporation, Fixed Point Theory and Applications, Volume 2006, Article ID 57950, Pages 1-13, DOI 10.1155/FPTA/2006/57950.

[11] A. Hajji, E. Hanebaly, Commutating mappings and $\alpha$-compact type fixed point theorems in locally convex spaces, Int. J. Math. Anal. 1 (2007), 661-680.

[12] Radouane Ziyad Azennar, Common fixed point theorems for single and multivalued mappings in complete ordered locally convex spaces. Math-Recherche et Application, Vol.16, (2017-2018), pp. 46-54

[13] Radouane Azennar, Fouad Ouzine, Driss Mentagui, Periodic point and fixed point results for monotone mappings in complete ordered locally convex spaces with application to differential equations, Advances in Fixed Point Theory, Vol 9, No 4 (2019), 322-332.

[14] Positive Operators, Charalambos D. Aliprantis and Owen Burkinshaw, Published by Springer,P.O. Box 17, 3300 AA Dordrecht, The Netherlands. 
[15] J. Yu, Essential equilibria of n-person noncooperative games, J. Math. Econom. 31 (1999), 361-372.

[16] R. Engelking, General Topology, 2nd ed., Sigma Series in PureMathematics, vol. 6, Heldermann, Berlin, 1989.

[17] Carl, S, Heikkilä, S: Fixed Point Theory in Ordered Sets and Applications: From Differential and Integral Equations to Game Theory. Springer, New York (2010)

[18] Ky Fan, Fixed-Point And Minimax Theorems In Locally Convex Topological Linear Spaces, Departement Of Mathematics, University Of Notre Dame Communicated by John von Neumann, December 13, 1951.

[19] Dhage, BC: A fixed point theorem for multi-valued mappings in ordered Banach spaces with applications I. Nonlinear Anal. Forum 10(1), 105-126 (2005)

[20] Nash, JF: Equilibrium points in n-person games. Proc. Natl. Acad. Sci. 36, 48-49 (1950)

[21] Debreu, G: A social equilibrium existence theorem. Proc. Natl. Acad. Sci. 38, 886-893 (1952)

[22] Granas, A, Dugundji, J: Fixed Point Theory. Springer, New York (2003) vol. 51, Academic Press, New York, 1968.

[23] Espaces topologiques, fonctions multivoques - Claude Berge

[24] Aubin, J.P., 1982. Mathematical Methods of Games and Economic Theory. North-Holland, Amsterdam.

[25] J.VonNeumann, Uber ein okonomisches Gleichungssystemund eine Verallgemeinerung des Brouwerschen Fixpunktsatzes, Ergebnisse eines Mathematischen Kolloquiums 8 (1937), 73-83 (German).

[26] H. Nikaid^o, Convex Structures and Economic Theory, Mathematics in Science and Engineering, vol. 51, Academic Press, New York, 1968.

[27] B. C. Dhage, A lattice fixed point theorem for multi-valued mappings with applications, Chinese J. Math., 19(1991), 11-22.

[28] J. Banas, K. Goebel, Measure of noncompactness in Banach spaces, 60 Dekker, New York, (1980). 